\title{
Exploring the effects of interventions to combat childhood obesity: inducing behavioural change in eating habits
}

\author{
Brian Dangerfield, Salford \\ Business School \\ University of Salford, Maxwell Building, \\ M5 4WT, United Kingdom \\ B.C.Dangerfield@salford.ac.uk
}

\author{
Norhaslinda Zainal Abidin, Salford Business \\ School \\ University of Salford, Maxwell Building, \\ M5 4WT, United Kingdom \\ N.ZainalAbidin@edu.salford.ac.uk
}

\begin{abstract}
The prevalence of English child obesity has increased especially in the 1990's. Due to the many negative impacts of obesity, such as the cost and health consequences, an urgent solution is needed to reverse current trends. This study focuses on behavioral changes looking at improvements in eating behavior. It is easier and sound logically to attempt changes in behavior compared to environmental changes which can be a costly and complicated process. Most of obesity research concerned with interventions is normally conducted in a real-life setting and using randomized controlled trials, but this demands time and cost. Due to these constraints, it is important to embrace other appropriate methods and computer simulation is one which offers considerable potential.
\end{abstract}

This study highlights the capability of system dynamics simulation modeling to evaluate obesity interventions looking at reversing the trends of weight, body mass index and the prevalence of obesity in children. Obesity is a complex problem in the real-life context. System dynamics has the capability to assess non-linear dynamic systems, which involve time delays and feedback processes and much useful information can be gleaned from a single analytical environment. Output runs from the modelling show that an increase in energy intake from food consumption is the main reason behind the increases in weight, body mass index and prevalence of obesity in a sample of English boys aged 11 to 15 years. Reducing the amount of energy derived from food consumption is a high leverage solution to the obesity problem.

Keywords- System dynamics, obesity, food intake, weight, body mass index, energy intake; energy expenditure

\section{INTRODUCTION}

The prevalence of child obesity has increased over time in worldwide pattern, particularly in developed countries in USA and Europe. In England, the prevalence of overweight and obese children has increased since 1995 (Health Survey for England, 2008). The split percentage of overweight and obese children aged 2 to 15 years in 1995 was $13.1 \%$ and $10.9 \%$ for boys and $13.1 \%$ and $12 \%$ for girls, increasing to $13.8 \%$ and $16.8 \%$ for boys and $14.1 \%$ and $16.1 \%$ for girls in 2007. If this trend continues, it is expected that around one quarter of the population aged below 20 could be obese in 2050 (Government Office for Science, 2007).
The causes of obesity are quite complex and they cannot be reduced to single factors. It is widely accepted that obesity occurs from over-eating behavior and a lack of enough physical exercise and it is supported by environments, medications and genetics that predispose to obesity conditions (Dehghan et al., 2005). Using an accounting statement analogy, people gain weight if there is an increase in energy intake or a fall in energy expenditure. Thus an increase in obesity will result from increased food intake and decreased exercise.

To overcome the problem, changes need to be made from a behavioral perspective. Behavioral change either by improving food intake and/or increasing physical activity (or both actions) have promised significant results in obesity prevention. Due to the importance of finding a solution from a behavioral changes perspective, the purposes for this paper are twofold: Firstly, to develop understanding (learning purpose) about how eating behavior contributes to obesity development. Secondly, to determine the most favourable interventions surrounding eating behavior change which strive to reverse trends in weight gain, BMI and the prevalence of obesity.

This paper is organized as follows: In the next section (section II), we discuss the system dynamics methodology that is used to model the child obesity problem. System dynamics (SD) is useful for modeling many complex problems in the health sector. In section III, we provide a theoretical framework to explain the scope, boundary and general structure for this work. This framework is used to guide our obesity work. In the fourth section (section IV), we provide some sample output runs from the model together with an explanation of how eating contributes to obesity development. It is followed by section $\mathrm{V}$ which reports on the results from behavioral change interventions experimented with in the model. The last section (section VI) provides a summary for the entire paper.

\section{SYSTEM DYNAMICS MODELLING}

Obesity is not only a complex but also a complicated problem due to two possible reasons (Levy et al., 2010). Firstly, many potential factors have roles in the development of obesity including environment, genetics, diet and the 
behavioural aspects of eating and physical activity (Government Office for Science, 2007). Secondly, obesity processes involve non-linear dynamics, time delays and feedback processes.

There is nothing particularly special about obesity. But it is recognized as a major medical issue and it provides many negative impacts on aspects of life. In general, obesity impacts can be observed from three important aspects: health risks, social problems, and financial cost consequences (Speiser et al., 2005). For children, obesity also impacts on their academic performance and ability to learn (Datar et al. 2004; Byrd et al. 2007).

Due to the many negative impacts of obesity, it is critically important to search for the interventions which might help to overcome the problem. Previous studies have discussed obesity work undertaken in the context of natural settings. This type of study is conducted with real people and in appropriate environmental settings and can provide a significant contribution towards obesity intervention (Harris et al. 2009; Sallis et al. 2003). Unfortunately, sometimes these methods are too costly and time consuming and produce no significant results in terms of weight and BMI reduction. Due to this disadvantage, it is important to search for another method which is less costly and can consider the obesity complexities and complications. Most of the methods which fulfil these criteria are based on a 'laboratory' or modelling background.

For obesity work, it is suggested that system dynamics simulation is one of the best options we have. The SD methodology was first devised by J. W. Forrester in 1950's (Forrester, 1961). It has been used by many researchers in understanding the behaviour of complex systems and obesity comes into that category. The advantage of SD is that realworld complexities can be experimented with by providing a framework for examining non-linear dynamics, time delayed effects, multiple interactions and feedback processes (Levy, 2010). Parallel to the objective of this work, that aims to understand the impact of the policy implications of behavioural changes, SD is also a good of choice of methodology due to its capability at the strategic level of policy making (Sterman, 2000).

System dynamics principles involve stock and flow concepts as shown in Figure A below.

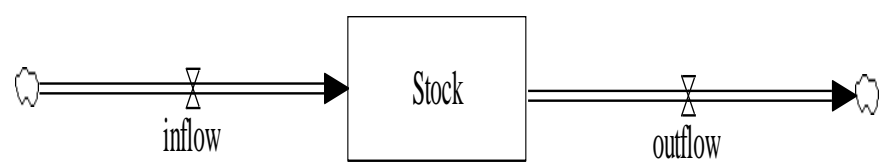

Figure A: Core diagram of a stock and two flows

A stock is an accumulation and changes to a stock can only come about by changing the inflow(s) and/or outflow(s) (Sterman, 2000). In this work, examples of stocks would be average weight; body mass index (BMI); and the prevalence of obesity (POO). These three important stocks are used to chart dynamic behaviour over 50 years. Inflows increase the size of the stock and outflows will decrease its value (Sterman, 2000). Because SD captures the changes in stock values over time, stocks need to be measured in units and flows in units/time. For example, weight, BMI and POO measured in $\mathrm{kg}$, $\mathrm{kg} /$ metre squared and a percentage respectively. For the flows, the units involve time (years) (e.g. kg/years, kg/metre $2 /$ years, and percentage/years).

\section{OBESITY FRAMEWORK}

In system dynamics modelling, it is important to provide the framework for the study being investigated (Sterman, 2000). This framework then can be used to explain the hypotheses underlying the work. It can also function as a guide to the modeller concerning the modelling process. Figure B is a framework used to explain the obesity hypothesis in this study. It considers, in basic terms, the most important variables in the model and depicts the scope and boundary of the model. This framework is called a sub-system diagram (Sterman, 2000) because it includes four different sectors which are connected to each other as follows: (1) Sector 1: food, (2) Sector 2: physical activity, (3) Sector 3: physical measurement, and (4) Sector 4: BMI Impact. In the overall process, each of these components is modelled differently and combined together to work as one component of the obesity system.

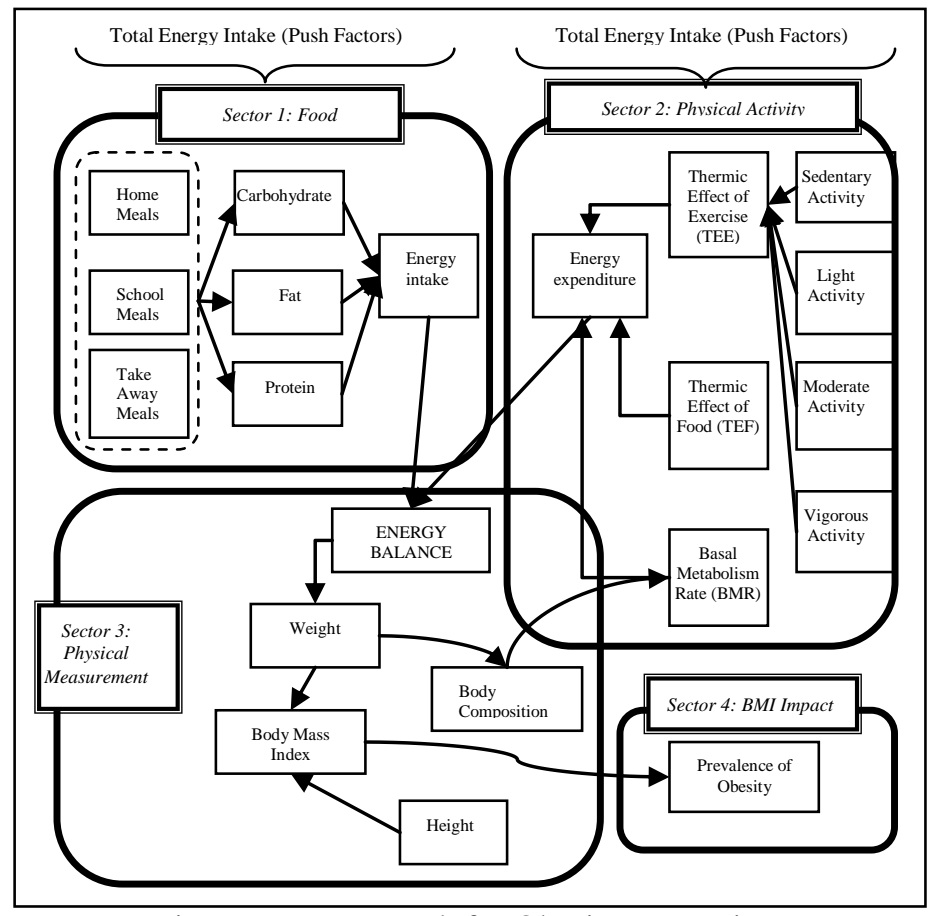

Figure B: Framework for Obesity Dynamics

The work attempts to explore the interactions between energy intake, energy expenditure and energy balance. Energy balance is the difference between energy intake and energy expenditure. Both physical activity and food intake energy expenditure are investigated. In the case of children, food intake is also needed for growth (not shown). A previous study by Egger and Swinburne (1997) stresses that obesity develops in three ways: (i) energy intake is greater than energy expenditure, (ii) energy expenditure is less than energy intake or (iii) both actions occur. 
Lambert and Goedecke (2003), Homer et al (2004; 2006) and Abdel-Hamid (2002; 2003) explain that obesity develops due to a feedback control between food intake, nutrient turnover, energy expenditure and body fat storage. Supported by this prior research, the model here attempts to chart obesity development from an energy intake, energy balance and energy expenditure perspective as follows: Changes in energy balance $=$ changes in energy intake - changes in energy expenditure

Changes in energy balance over time are the accumulated difference between energy intake and energy expenditure. If energy intake is greater than energy expenditure then the energy balance will increase; conversely if energy expenditure is higher than energy intake, then the energy balance will fall. If energy intake and energy expenditure are equal then the energy balance will be steady (Abdel-Hamid, 2002).

A daily positive energy balance is stored in muscle or fat. An increased body weight itself, however, generates higher energy expenditure, specifically energy from the basal metabolism rate. An equivalence between energy intake and energy expenditure (i.e. an equilibrium in energy balance) will ensure no weight change. The entire energy intake and expenditure processes can take years to show up as changes in average weight in the population (Homer et al, 2004). Our model is calibrated in years and so changes in daily energy balance and weight variability are smoothed out and this results in less sharp average weight changes in a year. However, because eating and performing physical activity is a daily process, the model additionally computes energy intake and energy expenditure in daily units (kcal/day) to aid understanding and to reflect common health metrics.

\section{MODEL DESCRIPTION}

Specifically, this work focuses on the English child population aged between 2 to 15 years for boys and girls. We have decided on this particular age group because the younger age is probably the best foundation to prevent obesity from progressing into adulthood (Dehghan et al, 2005). Furthermore, most of the interventions only work effectively for a specific age group and gender (Levy et al, 2000). Because of that, our model is divided into six different age groups and gender as follows: [2 to 4 years, Male], [2 to 4 years, Female], [5 to 10 years, Male], [5 to 10 years, Female], [11 to 15 years, Male], [11 to 15 years, Female]. The system dynamics software tool employed $\left(\right.$ Vensim $\left.^{\mathrm{TM}}\right)$ provides a subscript control tool that helps to perform this categorisation job.

Currently, our obesity model contains 28 (screen) views and 344 variables and parameter values. It has been validated by tests such as dimensional consistency, behaviour reproduction and extreme values (Sterman, 2000; Barlas, 1996; Forrester and Senge, 1980). The following section reports on sample results from the model.

\section{MODEL RESULTS}

The reported output graphs in this paper are only a part of our results. This section summarises the output obtained from the model (base case run) using three linked scenarios to describe the obesity process. The scenarios are as follows:

Scenario 1 (If energy intake $>$ energy expenditure=weight will increase): As long as a positive energy gap (=energy balance) is obtained in the form of energy intake being higher than energy expenditure, then weight is increased. In the opposite case, when the energy balance is falling (energy expenditure is more than energy intake) weight will decrease over time. Figure $\mathrm{C} 1$ below offers an example from a sample run (e.g. for the age group 11 to 15 years, male) to explain the above scenario. In contrast, Figure D1 demonstrates the case of energy intake being less than energy expenditure; the result is the opposite and weight decreases.

\section{Energy intake, energy expenditure and weight}

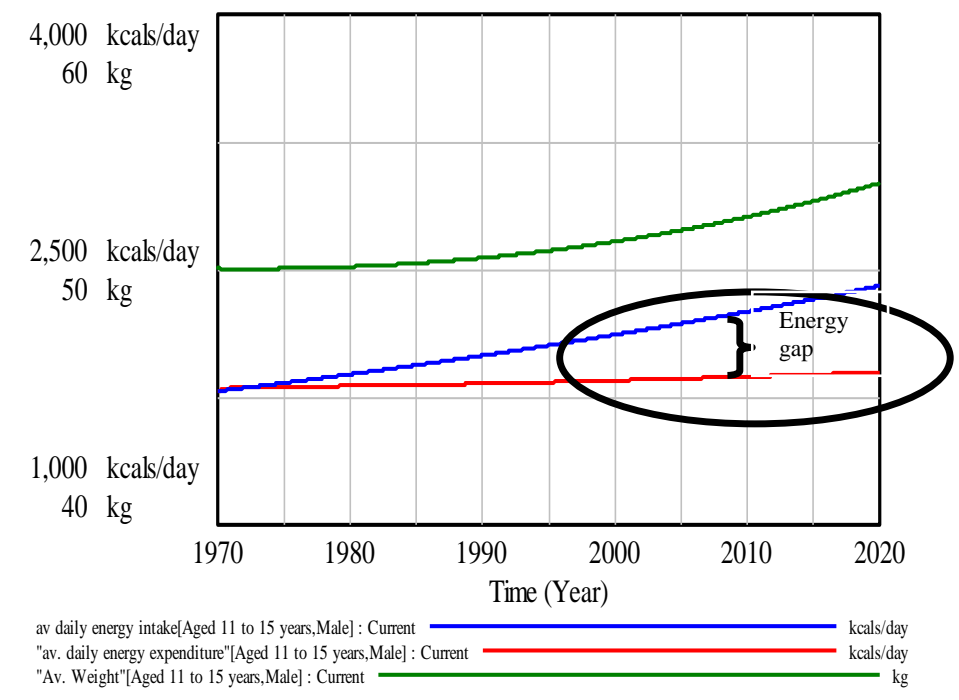

Figure $\mathrm{C} 1$ : Weight increase due to energy intake being higher than energy expenditure

Scenario 2 (If weight is increased and height is maintained then BMI increases): This scenario is applied in the situation when children collectively increase their weight over time. Height over the same period, as would be expected, shows little or no change. In these conditions, BMI should reflect an increase in value. Figure C2 provides an example of model output under this circumstance. The weight, height and BMI behaviour pattern is validated using the behaviour reproduction test (a comparison with realworld data). The data (Obesity Data [3]) is obtained from the Health Survey for England. This is an annual survey to monitor population health in England and it commenced in 1995 (Health Survey for England, 2008). 


\section{Weight, height and body mass index}

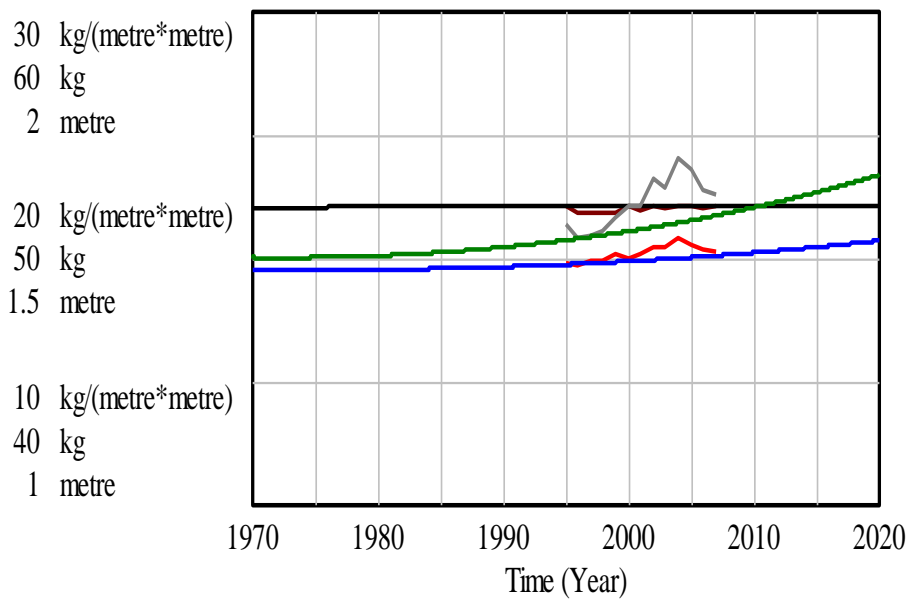

"Av. BMI"[Aged 11 to 15 years,Male] : Current

$\mathrm{kg} /$ (metre*metre)

"Av. BMI"[Aged 11 to 15 years,Male] : Obesity Data [3]

"Av. Weight"[Aged 11 to 15 years,Male] : Current

"Av. Weight"[Aged 11 to 15 years,Male] : Obesity Data [3]

"Av. Height"[Aged 11 to 15 years,Male] : Current

"Av. Height"[Aged 11 to 15 years,Male] : Obesity Data [3]

Figure C2: Increases in BMI value are reflected in weight gain

Scenario 3 (If BMI increases, the prevalence of obesity also increases): With increases in the BMI value, the prevalence of obesity also increases similarly as shown in Figure C3. A reduction in the average BMI value will result in a decrease in prevalence of obesity (see Figure D3).

\section{BMI and prevalence of obesity}

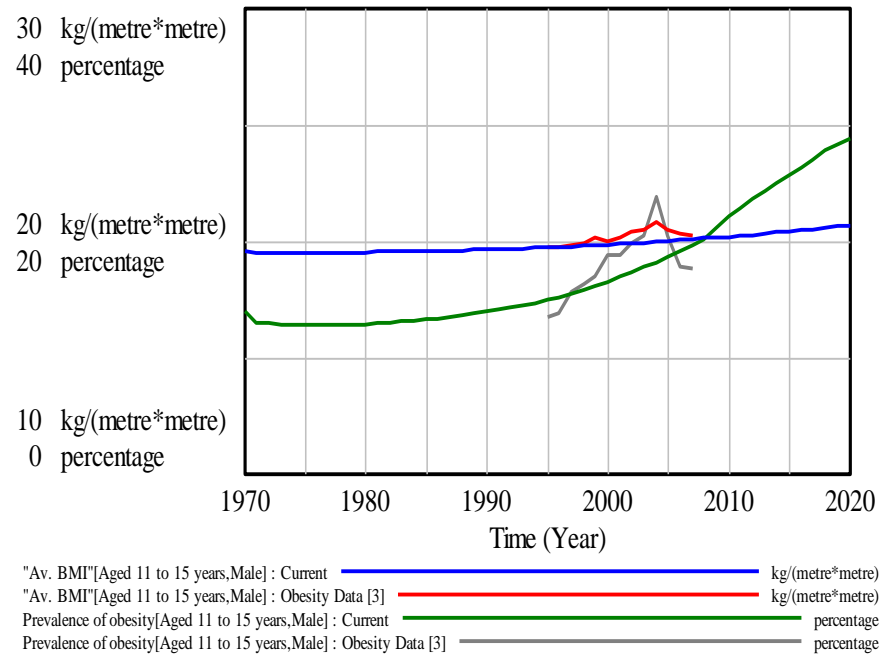

Figure C3: BMI and prevalence of obesity: an increasing behaviour pattern

All these scenarios have been tested using data on boys aged 11 to 15 years because of available historical data obtained from the Health Survey for England (2008). Using this data we have conducted a behaviour reproduction test to validate the consistency of the model. This test is useful for identifying flaws in model structure (Sterman, 2000).

Once confidence in the model has been developed, in terms of its structure and behaviour, then policy interventions can be conducted to identify what are the most effective policies that can be applied to reverse weight, BMI and prevalence of obesity trends. The next section discusses the interventions tested in the model.

\section{POLICY EXPERIMENTATION}

Research from previous studies has highlighted the importance of behaviour change to prevent obesity. One of the behavioural changes involves changes in eating behaviour. For the purpose of this paper, we focus only on modifications in eating behaviour since the primary aim of the paper is to understand how eating patterns contribute to the development of obesity. The results obtained from the model are divided into two different eating categories as follows:

Behaviour eating 1: (reduce the amount of energy intake and strive to maintain physical activity): The first eating behaviour change tested is to make a reduction in the intake of energy. In 1970, energy intake is only $1778 \mathrm{kcal} /$ day and this is reduced to $1607 \mathrm{kcal} / \mathrm{day}$ in 1990. However, the energy expenditure of $1778 \mathrm{kcal} /$ day has decreased to 1761 $\mathrm{kcal} /$ day in 1990. Nonetheless it is somewhat higher than energy intake. Figure D1 illustrates the decreasing behaviour pattern for both energy intake and energy expenditure between 1970 and 2020. We see that average weight falls from $50 \mathrm{~kg}$ in 1970 to $49.46 \mathrm{~kg}$ in 1990 . The decrease in weight occurs because of a negative energy balance condition, where energy expenditure is higher than energy intake.

Energy intake, energy expenditure and weight

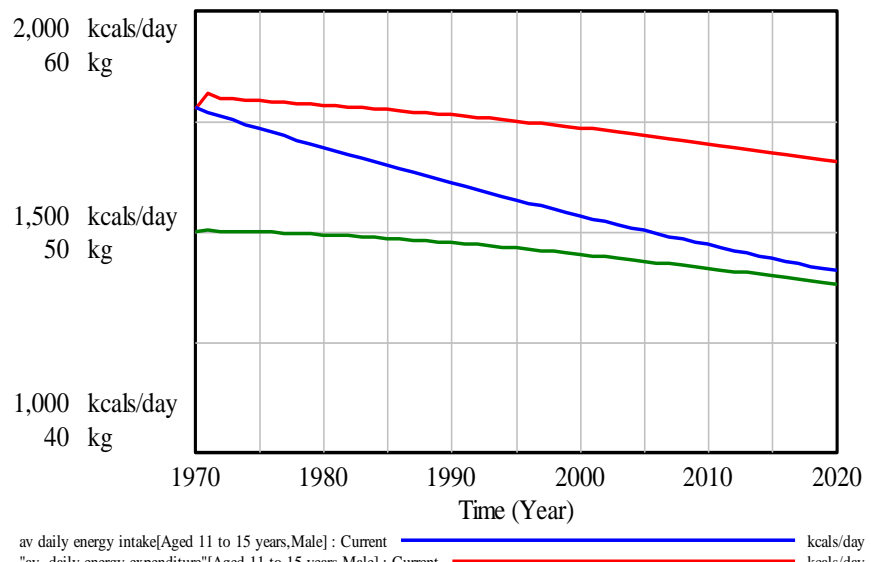

"av. daily energy expenditure"[Aged 11 to 15 years,Male] : Current $\mathrm{kcal} /$ /day

Figure D1: Impact of reducing energy intake on weight

Weight and height are correlated through the formula for BMI $[\mathrm{kg} /$ metre squared $]=[$ weight $/($ height $\times$ height $]$. With a reduction in weight and only a small increment in height it results in a fall in average BMI, as shown in Figure D2. Average BMI decreases from $19.50 \mathrm{~kg} / \mathrm{metre}^{2}$ to 18.44 $\mathrm{kg} / \mathrm{metre}^{2}$ between 1970 and 2020. With a decreasing BMI the prevalence of obesity follows a similar decreasing pattern as demonstrated in Figure D3. If a reduction in energy intake continues in this fashion, the prevalence of obesity is expected to fall from $12.9 \%$ in 1970 to $11.55 \%$ in 2020 . 
Weight, height and body mass index

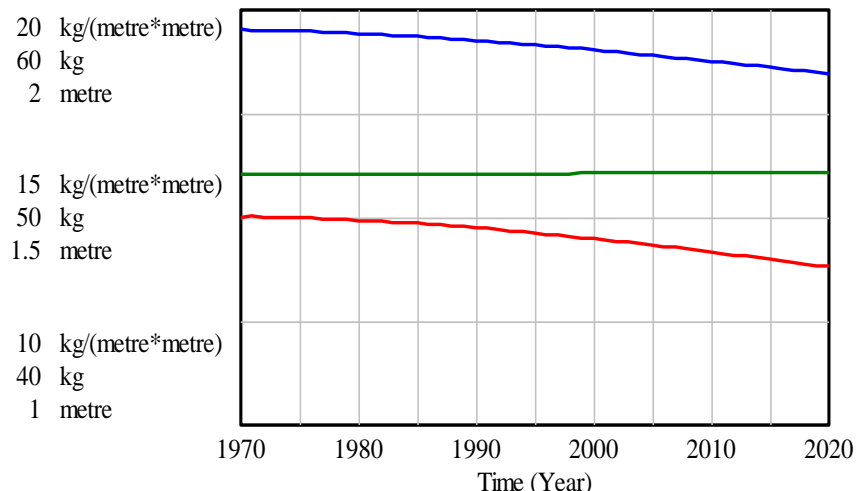

"Av. BMI"[Aged 11 to 15 years,Male]: Current $\longrightarrow \mathrm{kg} /($ metre*metre) "Av. Weight"[Aged 11 to 15 years,Male] : Current "Av. Height"[Aged 11 to 15 years,Male] : Current

Figure D2: Impact of decreasing weight on BMI

BMI and prevalence of obesity

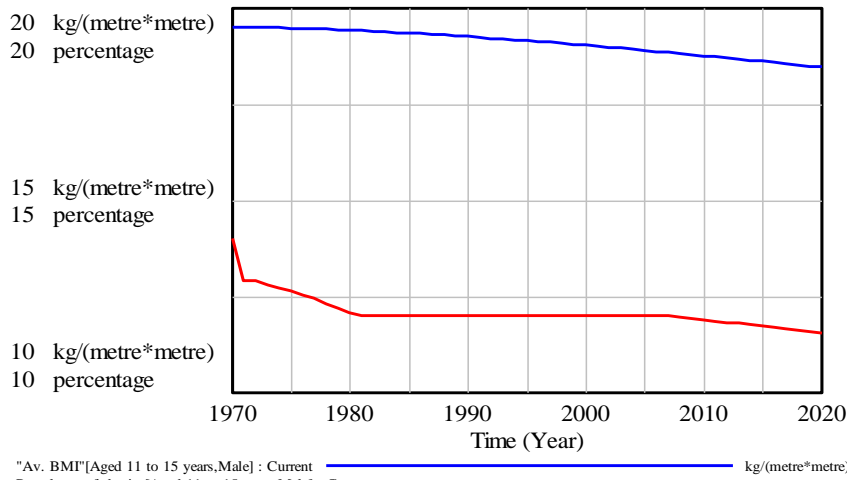

Figure D3: Impact of decreasing BMI on prevalence of obesity

Behaviour eating 2: (increase the amount of energy intake and maintain physical activity): In the second behaviour test we show the effect of increasing food consumption. For this context, energy intake is increased by $20 \%$ from the base case study. In 1970, an average English child consumed only $2134 \mathrm{kcal} /$ day. Now we have simulated the effects of energy intake increasing to $2384 \mathrm{kacal} /$ day in 1990 and to 2691 $\mathrm{kcal} / \mathrm{day}$ in 2010 . If there is no action taken to overcome this problem, then energy intake might reach, say, $2871 \mathrm{kcal} / \mathrm{day}$ in 2020 .

In the meantime, energy expenditure is also increased from $1776 \mathrm{kcal} /$ day in 1970 to $1964 \mathrm{kcal} /$ day in 2010 . It is suggested this value might increase to $2013 \mathrm{kcal} /$ day in 2020 . This increasing value for energy expenditure is due to the basal metabolism rate component which arises from the increase in weight. Figure E1 illustrates the increase in weight from 1970 to 2020 . Average weight of $50 \mathrm{~kg}$ in 1970 has increased to $51.10 \mathrm{~kg}, 52.47 \mathrm{~kg}, 54.12 \mathrm{~kg}, 56.10 \mathrm{~kg}$ and $58.44 \mathrm{~kg}$ in 1980, 1990, 2000, 2010 and 2020 respectively. The increase in weight is derived solely from a positive energy balance condition whereby children are eating more and not burning off this energy.
Energy intake, energy expenditure and weight

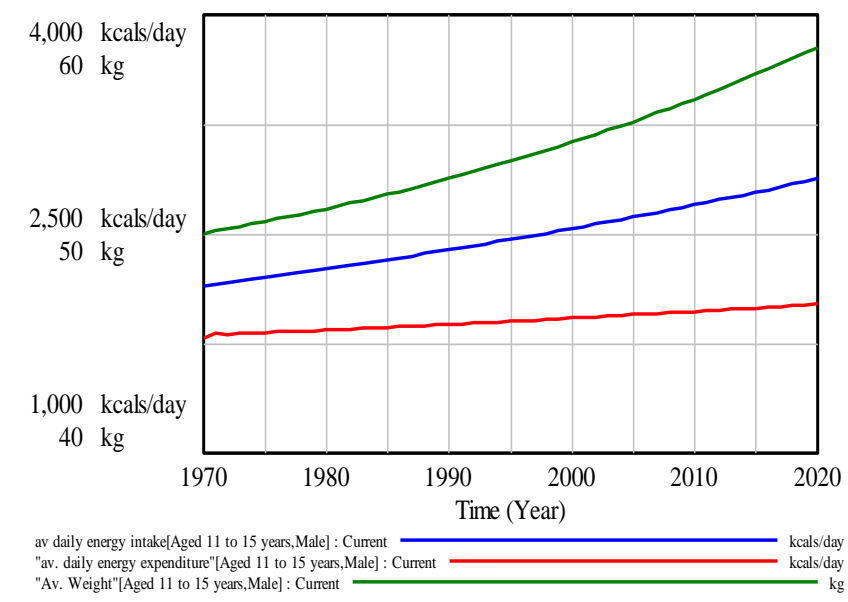

Figure E1: Impact of increasing energy intake on weight

In parallel to weight, increases in weight have also resulted in a similar increasing pattern in BMI. Average BMI of 19.5 $\mathrm{kg} / \mathrm{metre}^{2}$ in 1970 has increased to $21.77 \mathrm{~kg} / \mathrm{metre}^{2}$ in 2010 (see Figure E2). A similar pattern is observable for the prevalence of obesity. The prevalence of child obesity of $12 \%$ in 1970 is likely to have increased to $32.3 \%$ in 2000 and to $37.4 \%$ in 2020 (Figure E3).

\section{Weight, height and body mass index}

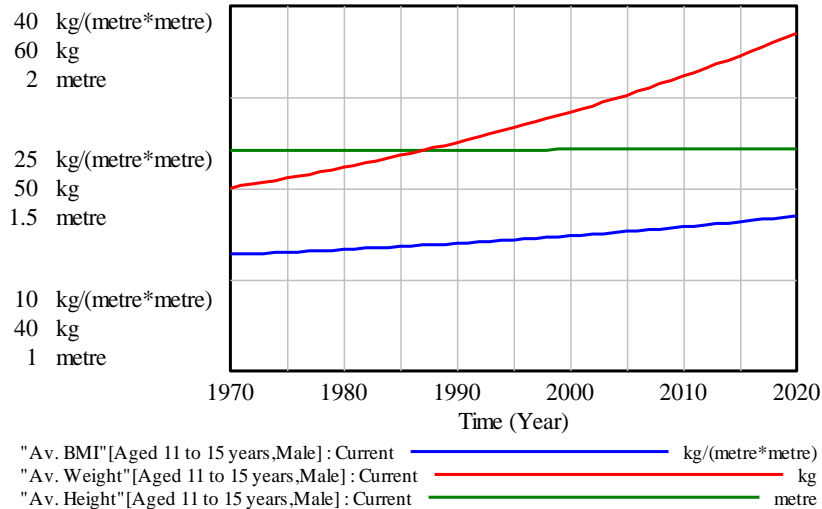

Figure E2: Impact of increasing weight on BMI

BMI and prevalence of obesity

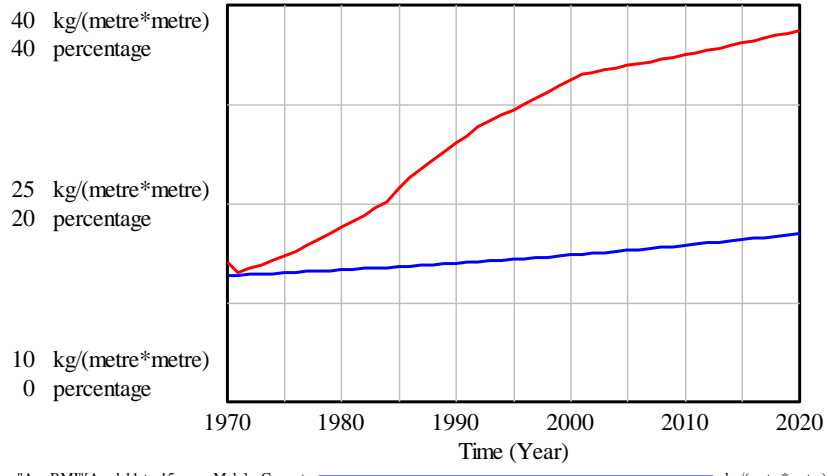

"Av. BMI"[Aged 11 to 15 years,Male] : Current 
Figure E3: Impact of increasing BMI on prevalence of obesity

\section{SUMMARY}

Obesity is the result of eating more and not burning off that extra energy. Obesity interventions through reducing the amount of energy intake provide significant positive results in terms of reversing weight gain, BMI rises and increases in the prevalence of obesity. System dynamics modeling is a tool that provides a platform to understand and to experiment with the contribution food consumption makes to obesity development. Output from the model runs highlight that an increase in the amount of energy intake results in increases in weight, BMI and prevalence of obesity in English children aged 11 to 15 years. In contrast, a reduction in the amount of energy intake supports positive results towards reducing weight, BMI and prevalence of obesity.

\section{FUTURE WORK}

This work is a part of ongoing research undertaken to evaluate behavioral changes that might counteract the obesity problem. We are expecting in future work to enhance the model. This will include:

i. More detail in modeling the food and physical activity sector. We are planning to base analysis upon the socio-psychological Theory of Planned Behavior (Azjen, 1991) to understand eating and physical activity decision processes. This socio-psychological theory highlights the personal elements in the decision making process and takes into consideration that surrounding forces and norms shape individual decisions.

ii. Together with this, we are planning to include environmental factors such as social norms and investigate how these norms play a role in influencing decision-making processes. Social norm interventions offer a new evidence-based approach for changing behavior which involves changing the perception about society's norms. It is difficult to maintain the same behavior if the supporting environment is not healthy and active.

\section{REFERENCES}

[1] Abdel-Hamid, T. K. (2002). Modelling the dynamics of human energy regulation and its implications for obesity treatment. System Dynamics Review, 18, 431-471.

[2] Abdel-Hamid, T, K. (2003). Exercise and diet in obesity treatment: an integrative system dynamics perspective. Journal of the American College of Sports Medicine, $35: 3,400-414$

[3] Ajzen, I. (1991). The theory of planned behaviour. Organizational Behaviour and Human Decision Processes, 50, 179-211.

[4] Barlas, Y. (1996). Formal aspects of model validity and validation in system dynamics. System Dynamics Review, 12:3, 183-210.
[5] Byrd, J. (2007). The impact of physical activity and obesity on academic achievement among elementary students. Available at http://cnx.org/content/m14420/latest/>

[6] Datar, A., Sturm, R. and Magnabosco, J. L. (2004). Childhood overweight and academic performance: National study of kindergarteners and first grades. Obesity Research, 12:1, 58-68.

[7] Dehghan, M., Akhtar-Danesh, N. And Merchant, A. T (2005). Childhood obesity, prevalence and prevention. Nutrition Journal, 4:24, 1-8.

[8] Egger, G. and Swinburn, B. (1997). An 'ecological' approach to the obesity pandemic. British Medical Journal, 315, 477-483.

[9] Forrester, J. W. (1961). Industrial Dynamics. Waltham, MA: Pegasus Communications

[10] Forrester, J. W. and Senge, P. M. (1980). Tests for building confidence in system dynamics models. TIMS Studies in Management Science, 14, 209-228.

[11] Government Office for Science (2007). Foresight Tackling Obesities: Future Choices <http://www.foresight.gov.uk/OurWork/ActiveProjects/ Obesity/KeyInfo/Index.asp $>[$ Accessed on 20 April 2009].

[12] Harris, K. C., Kuramoto, L. S., Schulzer, M. and Retallack, J. E. (2009). Effect of school-based physical activity interventions on body mass index in children: a meta analysis. Canadian Medical Association Journal, 31:7, 719-726.

[13] Health Survey for England (2008). Statistics on Obesity, Physical Activity and Diet: England 2008. The Department of Health. NHS England Report (2008). Statistics on Obesity, Physical Activity and diet: England, 2006: The Information Centre, 2006. Available at: $<\quad$ http://www.ic.nhs.uk/statistics-and-datacollections/health-and-lifestyles/obesity/ststistics-onobesity-physical-activity-and-diet england-2006>

[14] Homer J, Milstein, B, Dietz, W, Buchner D, and Majestic E (2006), Obesity population dynamics: exploring historical growth and plausible futures in the US. Procs of $24^{\text {th }}$ International System Dynamics Conference, July 2006, Nijmegen, July 2006.

[15] Homer J, Jones D, Milstein B, Murphy D, Essein J and Seville D (2004). Diabetes System Model Reference Guidance. Revised Edition.

[16] Lambert, E. V. and Goedecke, J. H. (2003). Energy balance and energy expenditure in obesity-is obesity is a disease of inactivity? Sports Medicine, Clinical Review.

[17] Levy, D. T., Mabry, P. L., Wang, Y. C., Gortmaker, S., Huang, T. T-K, Marsh, T., Moodie, M. and Swinburn, B. (2010). Simulation models of obesity: a review of the literature and implications for research and policy, Obesity Reviews, 1-17.

[18] Sallis, J. F., McKenzie, T. L., Conway, T. L., Elder, J. P., Prochaska, J. J., Brown, M., Zive, M. M., Marshall, S. J., and Alcaraz, J. E., (2003). Environmental interventions for eating and physical activity, a randomized controlled trial in middle school. American Journal of Preventive Medicine, 24:3, 209-217.

[19] Speiser, W., Rudolf, M. C. J., Anhalt, H., CamachoHubner, C., Chiarelli, F., Eliakim, A., Freemark, M., Gruters, A., Hershkovitz, E., Iughetti, L., Krude, H., Latzer, Y., Lustig, R. H., Pescovitz, O. H., PinhasHamiel, O., Rogol, A. D., Shalitin, S., Sultan, C., Stein, D., Vardi, P., Werther, G. A., Zadik, Z., ZuckermanLevin, N. and Hochberg, Z. (2005). Consensus statement: childhood obesity. The Journal of Clinical Endocrinology \& Metabolism, 90:3, 1871-1887.

[20] Sterman, J. D. (2000), Business dynamics: system thinking and modelling for a complex world. Boston, MA: Irwin McGraw-Hill. 